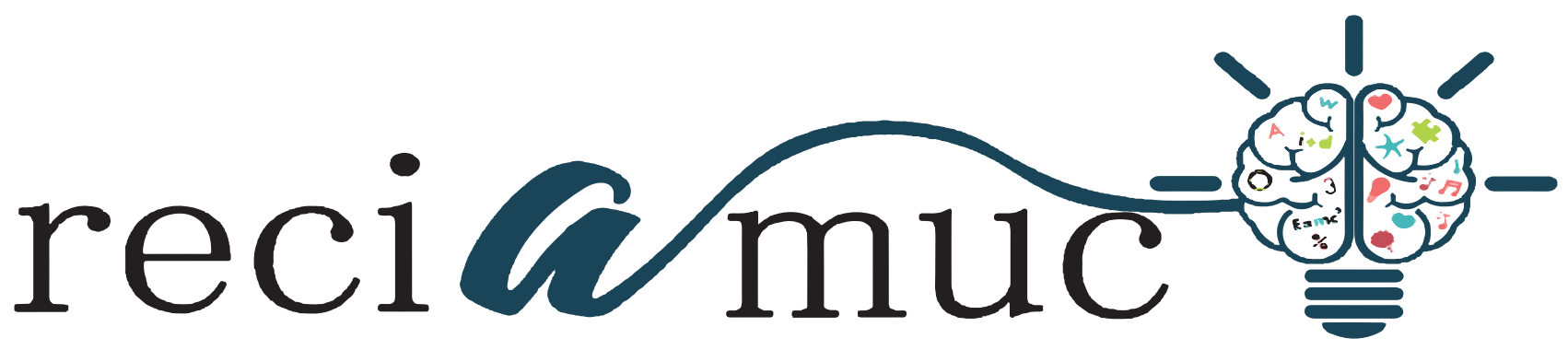

DOl: $10.26820 /$ reciamuc/4.(4).noviembre.2020.170-179

URL: https://reciamuc.com/index.php/RECIAMUC/article/view/552

EDITORIAL: Saberes del Conocimiento

REVISTA: RECIAMUC

ISSN: 2588-0748

TIPO DE INVESTIGACIÓN: Artículo de Revisión

CóDIGO UNESCO: 32 Ciencias Médicas

PAGINAS: $170-179$

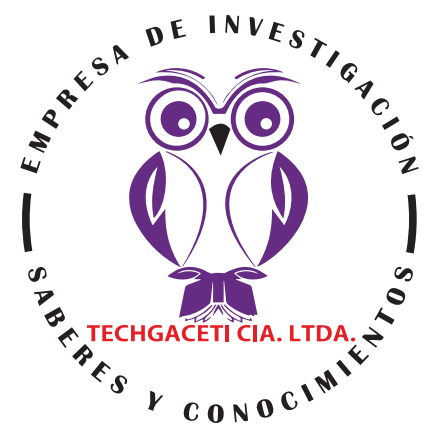

\title{
Tratamiento del síndrome de cushing iatrogénico: cuestiones de abstinencia de glucocorticoides
}

Treatment of iatrogenic cushing syndrome: glucocorticoid withdrawal issues Tratamento da síndrome do amortecimento iatrogénico: problemas de abstinência de glicocorticóides

Noemí Andrea Barrera Zambrano'; Andrea Isabel Ratti Torres²; Hilda Margarita Campoverde Chiquito3; Carlos Anthony Cárdenas Choez Correo ${ }^{4}$

RECIBIDO: 18/07/2020 ACEPTADO: 20/09/2020 PUBLICADO: 30/11/2020

1. Médico; Investigadora Independiente; Guayaquil, Ecuador; noemi_barrera15@hotmail.com; (iD https://orcid.org/00000002-7333-5149

2. Especialista en Medicina Interna; Médico; Investigadora Independiente; Guayaquil, Ecuador; (iD) andrearatti@hotmail.com; https://orcid.org/0000-0002-9053-9303

3. Médico; Investigadora Independiente; Guayaquil, Ecuador; hcampoverde1980@hotmail.com; (iD) https://orcid.org/00000001-6132-0620

4. Médico; Investigadora Independiente; Guayaquil, Ecuador; carlos-baterbass_91@hotmail.com; (D) https://orcid.org/00000002-1386-7741

CORRESPONDENCIA

María Verónica Crespo Zamora

noemi_barrera15@hotmail.com

Guayaquil, Ecuador

(c) RECIAMUC; Editorial Saberes del Conocimiento, 2020 


\section{RESUMEN}

El síndrome de Cushing iatrogénico es la forma más común de hipercortisolismo. Los glucocorticoides se usan ampliamente para el tratamiento de diversas enfermedades, a menudo en dosis altas que pueden conducir al desarrollo de hipercortisolismo severo. El hipercortisolismo iatrogénico es único, ya que la aplicación de glucocorticoides exógenos conduce a la presencia simultánea de síntomas específicos del hipercortisolismo y la supresión del eje endógeno hipotalámico-pituitario-adrenal. La función principal de su terapia está relacionada con el problema de la abstinencia de glucocorticoides. Existe una considerable variabilidad interindividual en la supresión y recuperación del eje hipotalámico-pituitario-adrenal, por lo tanto, La retirada y sustitución de glucocorticoides solo se puede realizar de manera escalonada con un seguimiento clínico cuidadoso y exámenes de laboratorio regulares con respecto a la actividad endógena del eje hipotalámico-pituitario-adrenal. Tres complicaciones principales que pueden asociarse con la abstinencia de glucocorticoides son: reactivación de la enfermedad subyacente, insuficiencia suprarrenal secundaria y síndrome de abstinencia de esteroides.

Palabras clave: latrogénico, hipercortisolismo, glucocorticoide, abstinencia, insuficiencia suprarrenal secundaria.

\section{ABSTRACT}

latrogenic Cushing syndrome is the most common form of hypercortisolism. Glucocorticoids are widely used for the treatment of various diseases, often in high doses that can lead to the development of severe hypercortisolism. Iatrogenic hypercortisolism is unique in that the application of exogenous glucocorticoids leads to the simultaneous presence of specific symptoms of hypercortisolism and suppression of the endogenous hypothalamic-pituitary-adrenal axis. The main issue of his therapy is related to the problem of glucocorticoid withdrawal. There is considerable inter-individual variability in suppression and recovery of the hypothalamic-pituitary-adrenal axis, therefore, glucocorticoid withdrawal and replacement can only be performed in a staggered manner with careful clinical follow-up and regular laboratory examinations regarding activity endogenous hypothalamic-pituitary-adrenal axis. Three main complications that can be associated with glucocorticoid withdrawal are: reactivation of the underlying disease, secondary adrenal insufficiency, steroid withdrawal syndrome.

Keywords: latrogenic, hypercortisolism, glucocorticoid, abstinence, secondary adrenal insufficiency.

\section{RESUMO}

A síndrome de Cushing iatrogénica é a forma mais comum de hipercortisolismo. Os glicocorticóides são amplamente utilizados para o tratamento de várias doenças, frequentemente em doses elevadas que podem levar ao desenvolvimento de hipercortisolismo grave. O hipercortisolismo iatrogénico é único na medida em que a aplicação de glicocorticóides exógenos leva à presença simultânea de sintomas específicos de hipercortisolismo e à supressão do eixo hipotalâmico-hipófise-adrenal endógeno. A questão principal da sua terapia está relacionada com o problema da retirada dos glicocorticóides. Há uma variabilidade considerável entre indivíduos na supressão e recuperação do eixo hipotálamo-hipófise-adrenal, portanto, a retirada e substituição do glicocorticóide só pode ser realizada de forma escalonada com um cuidadoso acompanhamento clínico e exames laboratoriais regulares relativamente à actividade hipotálamo-hipófise-adrenal endógena. Três complicações principais que podem ser associadas à retirada do glicocorticóide são: reactivação da doença subjacente, insuficiência adrenal secundária, síndrome de retirada de esteróides.

Palavras-chave: latrogénico, hipercortisolismo, glicocorticóide, abstinência, insuficiência adrenal secundária. 


\section{Introducción}

El síndrome de Cushing iatrogénico (exógeno) "es la forma de hipercortisolismo observada con mayor frecuencia en la práctica clínica" (Newell-Price \& Bertagna, 2006). Aunque generalmente da lugar a un cuadro clínico grave y puede aparecer tras el tratamiento de muchas enfermedades en las que se explotan los efectos antiinflamatorios, inmunosupresores e inductores de apoptosis de los glucocorticoides (por ejemplo, enfermedades autoinmunes, hematológicas, inflamatorias), su tratamiento sigue siendo controvertido. Mientras que en los últimos años se han publicado numerosos estudios sobre el tratamiento de otras formas de hipercortisolismo (formas espontáneas, endógenas), el tratamiento de las formas iatrogénicas solo se discute en una minoría de estudios.

Este síndrome es una afectación que se considera poco frecuente y que se presenta por lo general a los 25 y 40 años siendo más común en mujeres. Tomando en consideración que el cortisol es un tipo de hormona glucocorticoide producida por las dos glándulas suprarrenales y que las glándulas suprarrenales son pequeños órganos que se ubican sobre los riñones, cuando hay exceso de ésta hormona se considera que el paciente presenta síndrome Cushing lo cual se debe, muy probablemente, al exceso en la ingesta de drogas corticosteroides, como prednisona (síndrome de Cushing exógeno), o si el organismo produce demasiado cortisol, en este caso recibe el nombre de síndrome de Cushing endógeno.

El cuadro clínico del hipercortisolismo iatrogénico no es diferente al de las otras formas. Dado el cuadro clínico y los antecedentes de ingesta de glucocorticoides, su diagnóstico es sencillo. Como con frecuencia se aplican dosis elevadas y supra fisiológicas de glucocorticoides, el síndrome de Cushing iatrogénico suele ser muy severo. "La hipertensión es frecuente, pero la hipopotasemia es más rara que en las formas espontáneas, ya que los glucocorticoides sintéticos tienen una actividad mineralocorticoide más pobre en comparación con el cortisol natural" (Hopkins \& Leinung, 2005).

El punto más importante del tratamiento del síndrome de Cushing iatrogénico es el problema de la abstinencia de glucocorticoides. Retirar la terapia corta con glucocorticoides que dura algunas semanas o algunos meses como máximo, no conduce a problemas importantes en general. Sin embargo, retirar el tratamiento de glucocorticoides a largo plazo (varios meses o años) se convierte en un desafío tanto para el paciente como para el médico tratante

En el siguiente desarrollo investigativo se exponen las diferentes maneras de tratar este tipo de síndrome respecto a la abstinencia en la ingesta de glucocorticoides para pacientes que por alguna patología necesitan este tipo de drogas, en tal sentido, evaluar las posibilidades de tratamiento es el objetivo central de esta investigación en aras de brindar información necesaria y de interés para futuras investigaciones.

\section{Metodología}

Para el desarrollo de este proceso investigativo, se plantea como metodología la encaminada hacia una orientación científica particular que se encuentra determinada por la necesidad de indagar en forma precisa y coherente una situación, en tal sentido Davila, (2015) define la metodología "como aquellos pasos anteriores que son seleccionados por el investigador para lograr resultados favorables que le ayuden a plantear nuevas ideas" (p.66)

Lo citado por el autor, lleva a entender que el desarrollo de la acción investigativa busca simplemente coordinar acciones enmarcadas en una revisión bibliográfica con el fin de complementar ideas previas relacionadas Tratamiento del Síndrome de Cushing iatrogénico: cuestiones de abstinencia de glucocorticoides, a través de una revisión de literatura, para así finalmente elaborar un 
cuerpo de consideraciones generales que ayuden a ampliar el interés propuesto.

\section{Tipo de Investigación}

Dentro de toda práctica investigativa, se precisan acciones de carácter metodológico mediante las cuales se logra conocer y proyectar los eventos posibles que la determinan. En este sentido, la presente investigación corresponde al tipo documental, definido por Castro (2016), "se ocupa del estudio de problemas planteados a nivel teórico, la información requerida para abordarlos se encuentra básicamente en materiales impresos, audiovisuales y / o electrónicos". (p.41).

En consideración a esta definición, la orientación metodológica incluye la oportunidad de cumplir con una serie de actividades inherentes a la revisión y lectura de diversos documentos, donde se encuentran ideas explicitas relacionadas con los tópicos encargados de identificar una característica inmersa en el estudio. Por lo tanto, se realizaron continuas interpretaciones con el claro propósito de revisar aquellas apreciaciones propuestas por diferentes investigadores en relación al tema de interés, para luego dar la respectiva argumentación a los planteamientos, en función a las necesidades encontradas en la investigación, apoyados en las herramientas tecnológicas para la búsqueda de trabajos con valor científico disponibles en la web que tenían conexión con el objetivo principal de la investigación.

\section{Fuentes Documentales}

El análisis correspondiente a las características que predomina en el tema seleccionado, llevan a incluir diferentes fuentes documentales encargadas de darle el respectivo valor científico y en ese sentido cumplir con la valoración de los hechos a fin de generar nuevos criterios que sirven de referencia a otros procesos investigativos. Para Castro,(2016) las fuentes documentales incorporadas en la investigación documental o bibliográfica, "representa la suma de materiales sistemáticos que son revisados en forma rigurosa y profunda para llegar a un análisis del fenómeno" (p.41). Por lo tanto, se procedió a cumplir con la lectura previa determinada para encontrar aquellos aspectos estrechamente vinculados con el tema, con el fin de explicar mediante un desarrollo las respectivas apreciaciones generales de importancia.

\section{Técnicas para la Recolección de la Infor- mación}

La conducción de la investigación para ser realizada en función a las particularidades que determinan a los estudios documentales, tiene como fin el desarrollo de un conjunto de acciones encargadas de llevar a la selección de técnicas estrechamente vinculadas con las características del estudio. Bolívar, (2015), refiere, que es "una técnica particular para aportar ayuda a los procedimientos de selección de las ideas primarias y secundarias". (p.71).

Tal como lo expresa, Bolívar, (2015) "Las técnicas documentales proporcionan las herramientas esenciales y determinantes para responder a los objetivos formulados y llegar a resultados efectivos" (p. 58). Es decir, para responder con eficiencia a las necesidades investigativas, se introdujeron como técnica de recolección el método inductivo, que hizo posible llevar a cabo una valoración de los hechos de forma particular para llegar a la explicación desde una visión general. El autor Bolívar, (2015) tambien expresa que las técnicas de procesamiento de datos en los estudios documentales "son las encargadas de ofrecer al investigador la visión o pasos que deben cumplir durante su ejercicio, cada una de ellas debe estar en correspondencia con el nivel a emplear" (p. 123). Esto indica, que para llevar a cabo el procesamiento de los datos obtenidos una vez aplicadas las técnicas seleccionadas, tales como: fichas de resumen, textual, registros descriptivos entre otros, los mismos se deben ajustar al nivel que ha sido seleccionado. 


\section{Resultados}

\section{Preguntas sobre la abstinencia de la tera-} pia con glucocorticoides

Algunos autores argumentan que los tratamientos con glucorticoides de menos de tres semanas no suprimen realmente el eje sistema hipotálamo-pituitario-suprarrenal (HPA), independientemente de las dosis aplicadas, por lo que el tratamiento se puede interrumpir abruptamente sin una disminución gradual de la dosis. Sin embargo, otros datos muestran que la "supresión del eje HPA puede ocurrir incluso con tratamientos dosis altas de glucocorticoides tan cortos como 5 a 30 días, pero con dosis cercanas a niveles fisiológicos, la supresión clínicamente significativa es rara con tratamientos de menos de un mes" (Axelrod \& DeGroot, 2006).

El tratamiento con glucocorticoides inhibe a todos los participantes del eje HPA. La atrofia de la corteza suprarrenal ya se puede detectar incluso después de algunas semanas de tratamientos debido a la falta del efecto trófico de la adrenocorticotropina $(\mathrm{ACTH})$. Aunque la ACTH también influye un poco en el funcionamiento de la zona glomerulosa secretora de aldosterona.

El desarrollo de síntomas relacionados con el síndrome de Cushing muestra una fuerte correlación con la duración del tratamiento con esteroides: aunque no se espera el síndrome de Cushing después de una terapia con esteroides de cuatro semanas, la dosis de glucocorticoide debe retirarse gradualmente.

Cuanto mayor sea el tiempo de tratamiento con esteroides y mayor sea la dosis aplicada, mayor será el tiempo necesario para la abstinencia. En algunos casos, la regeneración del eje HPA lleva más de un año. Por otro lado, la reactividad del eje HPA puede preservarse con una dosis baja de esteroides, administrada por la mañana (por ejemplo, 5 mg o menos de prednisolona) (LaRochelle, 2003).
Es muy importante administrar esteroides por la mañana, ya que la supresión de la secreción de ACTH temprano en la mañana ocurre con menos frecuencia con este protocolo. El tipo de glucocorticoide también influye en el desarrollo de la supresión del eje HPA, ya que las preparaciones de mayor duración, por ejemplo, la dexametasona, suprimen la actividad HPA en mayor medida.

En vista de los graves efectos secundarios de la terapia con esteroides, se debe intentar administrar esteroides durante el período más breve posible y la retirada debe iniciarse lo antes posible. Si es necesaria la terapia crónica con esteroides, se puede recomendar la aplicación en días alternos después de algunas semanas de dosis iniciales más altas, ya que la supresión del eje HPA ocurre con menos frecuencia y la retirada es más fácil de realizar, en tal sentido, se debe intentar administrar esteroides durante el período más breve posible y la abstinencia debe iniciarse lo antes posible.

El problema más grave de la abstinencia de glucocorticoides "está relacionado con la alta variabilidad interindividual en cuanto al tiempo necesario para la supresión del eje HPA y la capacidad de su regeneración" (Charmandari, Nicolaides, \& George, 2014). La reactividad del hipotálamo y la pituitaria regresa antes que la de la corteza suprarrenal. El cuadro clínico y los exámenes de laboratorio, se pueden aplicar pruebas dinámicas para juzgar el proceso de regeneración.

\section{Pruebas para evaluar la actividad funcio- nal del eje HPA}

Es una pregunta difícil de responder cómo se ve la actividad funcional del eje HPA, o cuándo se puede omitir la terapia de sustitución con glucocorticoides. Aunque el cuadro clínico de insuficiencia suprarrenal (por ejemplo, mareos, hipotensión ortostática, náuseas, vómitos, hiponatremia, hipoglucemia, subfebrilidad, fiebre, confusión, dolor abdominal, shock, etc.) indica sin 
duda la insuficiencia del tratamiento, los parámetros objetivos son obligatorios. Para ello, "se pueden utilizar los niveles de cortisol matutinos obtenidos de sangre extraída entre las 6.00 y las 8.00 y pruebas dinámicas" (Charmandari, Nicolaides, \& George, 2014). Para la correcta interpretación tanto de los valores de cortisol matutinos como de las pruebas dinámicas, la sustitución de glucocorticoides debe suspenderse durante al menos 24 horas.

La fiabilidad de la prueba de laboratorio utilizada para determinar la concentración de cortisol es un punto importante. "Las sustancias esteroides pueden reaccionar de forma cruzada con el cortisol endógeno, según el protocolo de laboratorio utilizado, lo que podría alterar la interpretación de los resultados" (Vollenweider \& Waeber, 2013). Cuanto mayor sea la vida media del esteroide sintético, más tiempo se suspenderá su aplicación antes del examen.

Si el nivel de cortisol matutino es inferior a $3 \mu \mathrm{g} / \mathrm{dl}(85 \mathrm{nmol} / \mathrm{l})$, no se puede omitir la sustitución. "Niveles de cortisol superiores a $20 \mu \mathrm{g} / \mathrm{dl}$ (550 nmol / I) indican el correcto funcionamiento del eje HPA, en este caso se puede omitir la sustitución" (Hopkins \& Leinung, 2005). Las concentraciones de cortisol entre estos dos valores no son fiables como marcadores de la actividad del eje HPA, por lo que deben realizarse pruebas dinámicas.

Se encuentran disponibles varias pruebas dinámicas para evaluar la actividad funcional del eje HPA. Charmandari, Nicolaides, \& George, (2014) expresan:

Entre estos, la insulina-hipoglucemia $(\mathrm{IH})$ y la prueba de metirapona (MT) se utilizan para evaluar todo el eje HPA, la prueba de tetracosactida (Synacthen) se utiliza para el estudio de la corteza suprarrenal, mientras que la $\mathrm{CRH}$ (liberación de corticotropina hormona) -prueba se puede aplicar para examinar el subsistema de la corteza pituitaria-adrenal. (P. 67)
La prueba IH puede considerarse como el estándar de oro, ya que examina todo el eje HPA, además también es adecuada para estudiar otros sistemas hipotalámico-hipofisario, por ejemplo, la secreción de la hormona del crecimiento. "La hipoglucemia (glucosa en sangre $<2,2 \mathrm{mmol} / \mathrm{I}$ ) inducida con insulina regular de 0,1 a 0,15 U / kg de peso corporal activa el eje HPA" (Charmandari, Nicolaides, \& George, 2014).

Normalmente, la concentración sérica de cortisol debe superar los $20 \mu \mathrm{g} / \mathrm{dl}$ (550 nmol / I) en la muestra de sangre extraída durante la hipoglucemia. Los principales inconvenientes de la IHT son: es peligrosa debido al desarrollo de hipoglucemia, por lo tanto, no debe realizarse en ancianos y pacientes que padecen problemas cardíacos y epilepsia, consume mucho tiempo, es difícil y requiere vigilancia en un hospital.

La prueba de metirapona (MT) también es adecuado para estudiar todo el eje HPA.

La metirapona inhibe la enzima $11 \beta$-hidroxilasa que cataliza el paso final en la biosíntesis de cortisol, por lo que la producción reducida de cortisol induce la secreción de ACTH de la pituitaria. Como consecuencia de esto, aumentará la concentración sérica de ACTH y el precursor de cortisol 11-desoxicortisol (Compuesto S) (Fiad, Kirby, \& Cunningham, 2010).

Tras la ingesta de $30 \mathrm{mg} / \mathrm{kg}$ de metirapona entre las 23.00 y las 00.00 horas, "la concentración de 11-desoxicortisol en la muestra de sangre de la mañana siguiente debe ser superior a $7 \mu \mathrm{g} / \mathrm{dl}(200 \mathrm{nmol} / \mathrm{l})$, mientras que la concentración de cortisol debe ser inferior a 5 mg. / dl" (Fiad, Kirby, \& Cunningham, 2010). En algunas ocasiones, la MT puede provocar insuficiencia suprarrenal, sin embargo, es segura incluso para aplicaciones ambulatorias. "Su dificultad más grave está relacionada con la disponibilidad limitada de la medición del 11-desoxicortisol e incluso de la metirapona. Su sensibilidad es cercana a la de IHT" (Fiad, Kirby, \& Cunningham, 2010).

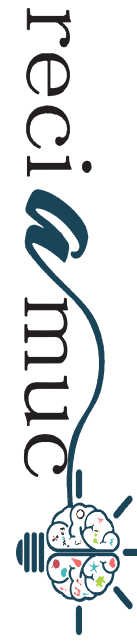


Es la prueba de tetracosactida corta (Synacthen) que se utiliza con mayor frecuencia para la evaluación del eje HPA. En caso de insuficiencia suprarrenal, "la concentración de cortisol sérico permanece por debajo de $20 \mu \mathrm{g} / \mathrm{dl}(550 \mathrm{nmol} / \mathrm{l}) 30$ minutos después de la inyección intravenosa de $250 \mu \mathrm{g}$ de tetracosactida (Synacthen, análogo de ACTH)" (Fiad, Kirby, \& Cunningham, 2010). Como la sensibilidad hacia la ACTH se reduce incluso después de algunos días después del cese de la secreción de ACTH, el Synacthentest (ST) no solo es adecuado para el diagnóstico de insuficiencia suprarrenal primaria sino también secundaria.

Por tanto, ST se puede aplicar para la evaluación de todo el eje HPA. Sin embargo, la "sensibilidad de ST es menor que la de IHT. Su sensibilidad puede mejorarse si la concentración de cortisol a superar se incrementa de $20 \mu \mathrm{g} / \mathrm{dl}$ (550 nmol / I) a $24 \mu \mathrm{g}$ / dl (650 nmol / I)" (Bangar \& Clayton, 2008), sin embargo, su especificidad puede verse reducida por esta modificación. Un problema significativo con el ST clásico es que la dosis estándar de $250 \mu \mathrm{g}$ de tetracosactida da como resultado una concentración sérica muchas veces mayor que el nivel fisiológico, por lo que puede no ser lo suficientemente sensible para el diagnóstico de formas más leves de insuficiencia suprarrenal secundaria.

En consecuencia, se aumentó la aplicación de una dosis más baja, es decir, 1 g (dosis baja de ST).

En sujetos sanos, $1 \mu \mathrm{g}$ de tetracosactida también produce concentraciones de cortisol superiores a $20 \mu \mathrm{g} / \mathrm{dl}(550 \mathrm{nmol} / \mathrm{l})$ 30 minutos después de la inyección. La utilidad de ST en dosis bajas en la insuficiencia suprarrenal secundaria es, sin embargo, polémico, por lo tanto, puede que no sea lo suficientemente sensible para el diagnóstico de formas más leves de insuficiencia suprarrenal secundaria. Por lo tanto, se aumentó la aplicación de una dosis más baja, es decir, 1 Hg (dosis baja de ST). (Charman- dari, Nicolaides, \& George, 2014)

Algunos autores sostienen que su sensibilidad alcanza la de las pruebas clásicas, mientras que otros informan que su sensibilidad y especificidad son mucho menores (Aguirre, Luna, \& Reyes, 2013). Un problema práctico con ST en dosis bajas está relacionado con el hecho de que el tetracosactid no está disponible en viales de $1 \mu \mathrm{g}$.

La prueba de hormona liberadora de corticotropina $(\mathrm{CRH})$ es adecuada para el estudio del subsistema pituitario-suprarrenal estimulando la adrenocorticotropina $(\mathrm{ACTH})$ y por lo tanto el cortisol.

Después de la inyección intravenosa de 1 $\mu \mathrm{g} / \mathrm{kg}$ de peso corporal de $\mathrm{CRH}$, se extrae sangre regularmente en un período de 2 horas (0, 15, 30, 60, 90 y 120 minutos). Si el funcionamiento pituitario y suprarrenal es normal, las concentraciones máximas de cortisol son superiores a $19 \mu \mathrm{g} / \mathrm{dl}$ (525 $\mathrm{nmol} / \mathrm{I})$. Esta prueba es segura y su sensibilidad es buena, pero su aplicación rutinaria está limitada por los altos costos de la $\mathrm{CRH}$ (Charmandari, Nicolaides, \& George, 2014).

\section{Las posibles consecuencias de la absti- nencia de glucocorticoides}

\section{Reactivación de la enfermedad subyacente}

La enfermedad subyacente puede reactivarse durante la abstinencia de glucocorticoides, lo que podría justificar la reintroducción de la terapia con glucocortoides. El paciente debe ser controlado regularmente durante la abstinencia e incluso después de su finalización. Debido a la posibilidad de reactivación, la retirada solo se puede realizar con la cooperación del médico que trata el trastorno subyacente y el endocrinólogo.

\section{Insuficiencia suprarrenal secundaria}

La insuficiencia suprarrenal secundaria es la complicación más temida de la abstinencia, ya que puede resultar en una afección aguda potencialmente mortal. Sin embargo, 
la insuficiencia suprarrenal rara vez se observa y, en general, se asocia con situaciones de estrés grave. En "contraste con los síntomas clásicos de la crisis de Addison, las alteraciones electrolíticas son poco frecuentes debido a la relativa preservación del sistema minero corticoide. Se requieren altas dosis de glucocorticoides parenterales para tratarlo" (Charmandari, Nicolaides, \& George, 2014).

\section{Síndrome de abstinencia de esteroides}

La patogenia de este síndrome es la menos aclarada entre las complicaciones asociadas con la abstinencia de glucocorticoides. Esta condición "se caracteriza por fenómenos inespecíficos que también pueden ocurrir en la insuficiencia suprarrenal, como náuseas, malestar, debilidad, fatiga, pérdida de peso, dolores articulares, subfebrilidad, fiebre, etc". (Hopkins \& Leinung, 2005). En contraste con las quejas y síntomas presentados, la sustitución de glucocorticoides es apropiada en esta condición y las pruebas dinámicas también dan resultados normales.

En el contexto de este síndrome se sospechan "mecanismos del sistema nervioso central (noradrenérgicos, sistemas dopaminerg, $\mathrm{CRH}$, péptidos opioides, prostaglandinas), que pueden ser similares a los procesos patológicos implicados en los síndromes de abstinencia de fármacos" (Axelrod \& DeGroot, 2006). Tales mecanismos parecen participar en los síndromes de abstinencia que se desarrollan después de suspender la administración de otras hormonas (estrógenos / menopausia, esteroides anabólicos) que pueden indicar la posibilidad de vías comunes. "Las citocinas inflamatorias, en su mayoría interleucina-6 liberadas en grandes cantidades, que se liberan de los efectos supresores de los glucocorticoides pueden estar involucradas en su patogenia" (Hochberg \& Pacak, 2003).

Algunos pacientes experimentan dependencia psicológica de los glucocorticoides que pueden complicar aún más la absti- nencia. "El síndrome de abstinencia de esteroides puede desarrollarse después de la recuperación del hipercortisolismo endógeno relacionado con la hipófisis o las suprarrenales" (Bhattachayya \& Kaushal, 2015). La dosis de sustitución de esteroides debe aumentarse transitoriamente para su tratamiento. Las citocinas inflamatorias, "en su mayoría interleucina-6 liberadas en grandes cantidades, que se liberan de los efectos supresores de los glucocorticoides pueden estar involucradas en su patogenia" (Vollenweider \& Waeber, 2013).

\section{Cómo retirar la terapia con glucocorticoides}

La abstinencia de glucocorticoides solo puede iniciarse "si la enfermedad subyacente se encuentra en una "fase inactiva de larga duración o en caso de enfermedad activa, si se introduce otra modalidad de tratamiento eficaz para suprimir la patología subyacente en lugar de glucocorticoides (por ejemplo otras formas de inmunosupresión) medicina presiva en enfermedades autoinmunes)" (Clevenbergh, Corcostegui, \& Gerard, 2010). La dosis del glucocorticoide aplicado debe reducirse a la dosis de sustitución característica del esteroide en particular (Tabla 1). Por tanto, puede iniciarse la abstinencia de glucocorticoides si la enfermedad subyacente se encuentra en una fase inactiva de larga duración con una dosis de esteroides correspondiente a la dosis de sustitución diaria o solo la sobrepasa ligeramente (por ejemplo, 4 a 6 mg de prednisolona / día). 
Tabla 1. Características más importantes de los glucocorticoides aplicados en la práctica clínica

Actividad

Vida media biológica antiinflamatoria Dosis de sustitución diaria en

Glucocorticoide (hora)

en relación al cortisol adultos $(\mathrm{mg})$

\begin{tabular}{lccc}
\hline Hidrocortisona & $<8-12$ & 1 & 20 \\
(cortisol) & $18-36$ & 4 & 5 \\
Prednisona & $18-26$ & 4 & 5 \\
Prednisolona & $18-36$ & 5 & 4 \\
Metilprednisolona & $18-36$ & 5 & 4 \\
Triamcinolona & $36-54$ & $25-30$ & $0,5-1$ \\
Dexametasona & & \\
\hline
\end{tabular}

Fuente: (Clevenbergh, Corcostegui, \& Gerard, 2010)

Se observa con frecuencia que los pacientes refieren quejas correspondientes al síndrome de abstinencia de esteroides, aunque la dosis de sustitución es adecuada. En este caso, la dosis de glucocorticoides debe aumentarse transitoriamente según el cuadro clínico, luego, paralelamente a la mejora de los síntomas, se puede intentar nuevamente la abstinencia.

\section{Conclusiones}

Durante el desarrollo de la investigación se observó, que no existe consenso sobre el protocolo de retirada de esteroides. Se puede afirmar que la abstinencia solo se puede realizar de forma gradual, reduciendo las dosis de glucocorticoides de forma escalonada. Hay dos puntos de vista principales con respecto al tipo de glucocorticoide aplicado. Uno propone la introducción de un régimen de ingesta de esteroides en días alternos, sin cambiar la medicación glucocorticoide utilizada anteriormente. Este cambio en la ingesta, no puede ser abruptamente al día alterno, ya que los síntomas de insuficiencia suprarrenal pueden aparecer en los días sin el esteroide.

El otro punto de vista sugiere el uso de hidrocortisona en lugar de otros glucocorti- coides sintéticos utilizados anteriormente. La hidrocortisona, que tiene la misma estructura química que el cortisol endógeno, suprime el funcionamiento del eje HPA en menor grado que otros glucocorticoides sintéticos. Debido a su vida media biológica más corta, la hidrocortisona disminuye los síntomas del síndrome de Cushing, por otro lado, debe administrarse al menos dos veces al día.

Sin embargo, el diagnóstico y tratamiento del hipercortisolismo sigue siendo uno de los problemas más rebuscados de la endocrinología clínica. Las formas iatrogénicas (exógenas) de hipercortisolismo son mucho más frecuentes que las endógenas a pesar de que la literatura que lo trata sigue siendo escasa en relación con las otras manifestaciones. En tal sentido y en contraste con su destacada relevancia clínica, no existe una guía generalmente aceptada para el protocolo de abstinencia de glucocorticoides.

Por esta razón, debido a la gran variación interindividual, el protocolo y el calendario de retirada de glucocorticoides solo se pueden determinar individualmente, con el examen paralelo del cuadro clínico y los resultados de laboratorio. Como complicaciones graves, es decir, reactivación de la 
enfermedad subyacente, pueden aparecer insuficiencia suprarrenal secundaria y síndrome de abstinencia de esteroides durante la abstinencia de glucocorticoides.

\section{Bibliografía}

Aguirre, M., Luna, M., \& Reyes, Y. (2013). Diagnóstico y Manejo de la Insuficiencia Adrenal. Revista Venezolana Endocrinologia Metab, 11(3), 157167.

Axelrod, L., \& DeGroot, L. (2006). Glucocorticoid therapy. Endocrinology, 2329-2342.

Bangar, V., \& Clayton, R. (2008). How reliable is the short synacthen test for the investigation of the hypothalamic-pituitary-adrenal axis? Eur. J. Endocrinol, 580-583.

Bhattachayya, A., \& Kaushal, K. (2015). Steroid withdrawal syndrome treatment of Cushing's syndrome: a reminder. Eur. J. Endocrinol, 207-210.

Charmandari, E., Nicolaides, N., \& George, P. (25 de Mayo de 2014). Glucocorticoid-induced adrenal insufficiency. Chrousos Lancet, 383, 67-152.
Clevenbergh, P., Corcostegui, M., \& Gerard, D. (2010). latrogenic Cushing's syndrome in an HIV infected patient treated with inhaled corticosteroids (fluticasone propionate) and low-dose ritonavir enhanced PI containing regimen. J. Infect, 54.

Fiad, T., Kirby, J., \& Cunningham, S. (2010). The overnight single-dose Metopyrone test is a simple and reliable index of the hypothalamic-pituitary-adrenal axis. . Clin. Endocrinol, 603-609.

Hochberg, Z., \& Pacak, K. (2003). Endocrine withdrawal syndromes. Endocr. Rev, 523-538.

Hopkins, R., \& Leinung, M. (2005). Exogenous Cushing's syndrome and glucocorticoid withdrawal. Endocrinol. Metab Clin. North. Am, 371-384.

LaRochelle, G. (2003). Recovery of the hypothalamic-pituitary-adrenal (HPA) axis in patients with rheumatic diseases receiving low-dose prednisone. Am. J. Med, 258-264.

Newell-Price, J., \& Bertagna, X. (2006). Cushing's syndrome. Lancet, 1605-1617.

Vollenweider, P., \& Waeber, G. (2013). Planifier un sevrage aux glucocorticoïdes: stratégie diagnostique et thérapeutique. Revue Praxis, 1675-1682.

\section{CITAR ESTE ARTICULO:}

Jimenez, N. A. B. Z., Ratti Torres, A. I., Campoverde Chiquito, H. M., \& Cárdenas Choez, C. A. (2020). Tratamiento del síndrome de cushing iatrogénico: cuestiones de abstinencia de glucocorticoides. RECIAMUC, 4(4), 170-179. https://doi.org/10.26820/reciamuc/4.(4).noviembre.2020.170-179 